\title{
Adaptive Fuzzy Tracking Control of Rotation Speed for Macro Gas Turbine
}

\author{
Wei Deng ${ }^{1, \mathrm{a}^{*}}$, Yang Ma ${ }^{1, \mathrm{~b}}$ and Bing Zhang ${ }^{2, \mathrm{c}}$ \\ ${ }^{1}$ Department of automatic control, Shenyang Institute of Engineering, Shenyang 110136, China \\ ${ }^{2}$ College of Science, Shenyang Agricultural University, Shenyang 110866, China \\ adw0310204@126.com, bmayanghc@163.com, 'zhangbsy@163.com
}

Keywords: Gas turbine; Fuzzy control; Rotation speed control; Nonlinear system; Tracking control

\begin{abstract}
Macro gas turbines (GT) are a newly developed small heat engine. GT is a whole by a combination of components such as turbine, combustor. It is a class of heat engines, which transform heat energy into mechanical energy. It is also a complicated thermo dynamical and mechanical system that cannot be modeled accurately. The mathematical model of a gas turbine is complex and very strong nonlinear. In this paper, an adaptive fuzzy control strategy is proposed for the gas turbine, which belongs to a class of nonlinear systems involving plant uncertainties. A robust control compensator is derived to eliminate the effect of uncertainties. The results of simulation demonstrate that the effect of the external disturbance on the rotation speed error can be attenuated to prescribed level and then the rotation speed control performance is achieved. It is proved that the rotation speed tracking error can converge to a small neighborhood of origin and that all the states and signals of the closed-loop system are ultimately uniformly bounded.
\end{abstract}

\section{Introduction}

Macro gas turbines have a very important position in the industrial and military fields. Fuzzy control has been successfully used to many control problems because it needs no accurate mathematical models of the controlled plants[1,2]. Therefore, the researches on fuzzy adaptive control methods of gas turbines are significant in academic and practical aspects.

There are two kinds of uncertainties in a system to be controlled. One is caused by a lack of information about system structure and parameters, and the other is due to internal and external disturbances. Because of these uncertainties, the conventional nonlinear methods like feedback linearization usually fail, and the control of nonlinear uncertain systems remains a challenging. The objective, in adaptive control, is to introduce an adaptation law that adjusts the parameters of the controller or the system against system uncertainties and disturbances $[3,4]$.

Design of tracking controller has broad application background, and now it has made a number of research results [5,6,7]. Fuzzy control with good use of a priori knowledge of human does not rely on a precise mathematical model of the plant. It has a lot of application development in the tracking control of nonlinear systems. The paper for a class of SISO uncertain nonlinear systems of macro gas turbine has proposed the adaptive fuzzy state feedback tracking control strategy, and given stability analysis.

\section{Description of the Fuzzy System}

Consider the affine SISO nonlinear system as follows.

$$
y^{(r)}=F(x)+G(x) u+d
$$

where $x=\left(x_{1}, x_{2}, \cdots, x_{n}\right)^{\mathrm{T}} \in R^{n}$ for the measurable state vector. In the controlled area of $x, G(x) \neq 0$.

If some of the priori knowledge of human can be described by following IF-THEN rules:

$\widehat{R}$ : IF $x_{1}$ is $A_{1}$ and $\cdots$ and $x_{n}$ is $A_{n} \operatorname{THEN} u$ is $\widehat{B}_{n}$ 
The fuzzy sets $A \in \mathrm{R}, \widehat{B}_{n} \in \mathrm{R}$, then the single fuzzy controller is chosen as $u=u(x \mid \xi) . \xi$ is the adjustable parameter sets. Fuzzy system $u(x \mid \xi)$ is constructed as following fuzzy rules:

$\widetilde{R}$ : IF $x_{1}$ is $C_{1}$ and $\cdots$ and $x_{n}$ is $C_{n}$ THEN $u$ is $\widetilde{D}$

So the fuzzy system can be written as follow, where, $\mu(x)$ is the fuzzy basis function.

$$
u(x \mid \xi)=\mu^{T}(x) \xi=\xi^{T} \mu(x)
$$

\section{Design of Fuzzy Controller}

Let $e=y_{m}-y, \hat{e}=\left(e, \dot{e}, \cdots, e^{(r-1)}\right)^{T}$, where $\hat{e}$ is the error vector. $y_{m}$ is the given reference input signal. If the functions $F(x), G(x)$ are known, the ideal control

$$
\bar{u}=\frac{1}{G(x)}\left(K^{T} \hat{e}-F(x)+y_{m}^{(r)}\right)
$$

can make the system Eq.1 into a linear and controllable.

The control law is chosen as follows[8]

$$
u=u(x \mid \xi)-u_{R} / G(x)
$$

where $u_{R}$ for the robust $H_{\infty}$ control. Substituting Eq.4 into Eq.1, we get

$$
\begin{aligned}
& y^{(r)}=F(x)-u_{\mathrm{R}}+G(x) u(x \mid \xi)+d \\
& F(x)=-G(x) \bar{u}+K^{T} \hat{e}+y_{\mathrm{m}}^{(r)}
\end{aligned}
$$

Substituting Eq.8 into Eq.7, we have

$$
\begin{aligned}
& e^{(r)}=-G(x)(u(x \mid \xi)-\bar{u})+u_{\mathrm{R}}-K^{T} \hat{e}-d \\
& \dot{\hat{e}}=T \hat{e}+t u_{\mathrm{R}}-t G(x)(u(x \mid \xi)-\bar{u})-t d
\end{aligned}
$$

$T, t$ are defined as $T=\left(\begin{array}{ccccc}0 & 1 & 0 & \cdots & 0 \\ 0 & 0 & 1 & \cdots & 0 \\ \vdots & \vdots & \vdots & \ddots & \vdots \\ -k_{r} & -k_{r-1} & -k_{r-2} & \cdots & -k_{1}\end{array}\right), t=\left(\begin{array}{llll}0 & 0 & \cdots & 1\end{array}\right)^{\mathrm{T}}$.

The optimal parameters of vector $\xi$ and the minimum approximation error of $u(x \mid \xi)$ is defined as follows, respectively, $\xi^{*}=\underset{\xi \in \hat{R}}{\operatorname{argmin}}\left(\sup _{x \in R^{n}}\|u(x \mid \xi)-\hat{u}\|\right), u^{\prime}=\left(\hat{u}-u\left(x \mid \xi^{*}\right)\right) G(x)$.By Eq.8 and Eq.2, it get

$$
\begin{aligned}
& \dot{\hat{e}}=T \hat{e}-t\left(\mu^{T}(x)\left(\xi-\xi^{*}\right)\right) G(x)+t u^{\prime}+t u_{\mathrm{R}}-t d \\
& \dot{\hat{e}}=T \hat{e}-t G(x) \mu^{T}(x) \tilde{\xi}+t u_{\mathrm{R}}+t \varepsilon
\end{aligned}
$$

where $\tilde{\xi}=\xi-\xi^{*}, \varepsilon=u^{\prime}-d$. Here, the control target is to design fuzzy control law $u_{l}=u(x \mid \xi)$ and the adaptive law of parameters vector $\xi$ to make the control system stable globally and can achieve the following $H_{\infty}$ performance.

$$
\int_{0}^{t_{\mathrm{f}}} \hat{e}^{\mathrm{T}} Q \hat{e} d t \leq \hat{e}^{\mathrm{T}}(0) P \hat{e}(0)+\frac{1}{\alpha} \tilde{\xi}^{T}(0) \tilde{\xi}(0)+\beta^{2} \int_{0}^{t_{\mathrm{f}}} \varepsilon^{T} \varepsilon \quad, \quad t_{\mathrm{f}} \in[0, \infty), \varepsilon \in L_{2}\left[0, t_{\mathrm{f}}\right]
$$

the matrix $P^{\mathrm{T}}=P>0, Q^{\mathrm{T}}=Q>0$ and the given parameters $\alpha>0, \beta>0$.

Consider system Eq.1, if control law $u_{l}$ is chosen as Eq.12, adaptive law of parameter vector $\xi$ is Eq.13 , robust compensation control $u_{R}$ is Eq.14, then the closed-loop system can satisfy the following control performance: (1) For $\forall t \in[0, \infty)$, we have $u_{l}, e(t), x(t) \in L_{\infty}$, respectively; (2) For prescribed level $\beta>0$, control system can reach the $H_{\infty}$ tracking performance of Eq.11.

$$
\begin{aligned}
& u_{l}=\mu^{T}(x) \xi-u_{\mathrm{R}} / G(x) \\
& \dot{\xi}=\alpha \mu(x) t^{\mathrm{T}} P \hat{e} G(x) \\
& u_{\mathrm{R}}=-\gamma \hat{e}
\end{aligned}
$$


The definite matrices $Q, P$ are the solutions of following linear matrix inequality

$$
P T+T^{\mathrm{T}} P-\gamma^{\mathrm{T}} t^{\mathrm{T}} P-P t \gamma+\frac{1}{\sigma^{2}} P t t^{\mathrm{T}} P+4 Q-2 \tau I<0
$$

$\tau>0$ is an arbitrary constant.

Proof Lyapunov function is chosen as follows

$$
V=\frac{1}{2} \hat{e}^{\mathrm{T}} P \hat{e}+\frac{1}{2 \alpha} \tilde{\xi}^{T} \tilde{\xi}
$$

Duo to $\dot{\tilde{\xi}}=\dot{\xi}$, then

$$
\dot{V}=\frac{1}{2} \dot{\hat{e}}^{\mathrm{T}} P \hat{e}+\frac{1}{2} \hat{e}^{\mathrm{T}} P \dot{\hat{e}}+\frac{1}{2 \alpha} \dot{\tilde{\xi}}^{T} \tilde{\xi}+\frac{1}{2 \alpha} \tilde{\xi}^{T} \dot{\vec{\xi}}=\frac{1}{2} \dot{\hat{e}}^{\mathrm{T}} P \hat{e}+\frac{1}{2} \hat{e}^{\mathrm{T}} P \dot{\hat{e}}+\frac{1}{\eta_{\mathrm{d}}} \tilde{\xi}^{T} \dot{\xi}
$$

Substituting Eq.10 into Eq.17, we have

$$
\dot{V}=\frac{1}{2}\left(\hat{e}^{\mathrm{T}} T^{\mathrm{T}}-\tilde{\xi}^{\mathrm{T}} \mu(x) t^{\mathrm{T}} G(x)-\hat{e}^{\mathrm{T}} \gamma^{\mathrm{T}} t^{\mathrm{T}}+\varepsilon^{T} t^{\mathrm{T}}\right) P \hat{e}+\frac{1}{2} \hat{e}^{\mathrm{T}} P \times\left(T \hat{e}-t G(x) \mu^{T}(x) \tilde{\xi}-t \gamma \hat{e}+t \varepsilon\right)+\frac{1}{\alpha} \tilde{\xi}^{T} \dot{\xi}
$$

Noting that $\tilde{\xi}^{T} \mu(x) t^{\mathrm{T}} G(x) P \hat{e}=\hat{e}^{\mathrm{T}} \operatorname{Pt} G(x) \mu^{T}(x) \tilde{\xi}$, then we get

$$
\dot{V}=\frac{1}{2} \hat{e}^{\mathrm{T}} P T \hat{e}+\frac{1}{2} \hat{e}^{\mathrm{T}} T^{\mathrm{T}} P \hat{e}-\frac{1}{2} \hat{e}^{\mathrm{T}} \gamma^{\mathrm{T}} t^{\mathrm{T}} P \hat{e}-\frac{1}{2} \hat{e}^{\mathrm{T}} P t \gamma \hat{e}+\frac{1}{2} \varepsilon^{\mathrm{T}} t^{\mathrm{T}} P \hat{e}+\frac{1}{\alpha} \tilde{\xi}^{\mathrm{T}}\left(\dot{\xi}-\alpha G(x) \mu(x) t^{\mathrm{T}} P \bar{e}\right)+\frac{1}{2} \hat{e}^{\mathrm{T}} P t \varepsilon
$$

Let $\dot{\xi}=\alpha G(x) \mu(x) t^{\mathrm{T}} P \hat{e}$, then Eq.19 can be written as

$$
\dot{V}=\frac{1}{2} \hat{e}^{\mathrm{T}}\left(P T+T^{\mathrm{T}} P-\gamma^{\mathrm{T}} t^{\mathrm{T}} P-P t \gamma\right) \hat{e}+\frac{1}{2}\left(\varepsilon^{T} t^{\mathrm{T}} P \hat{e}+\hat{e}^{\mathrm{T}} P t \varepsilon\right)
$$

Duo to $\varepsilon^{T} t^{\mathrm{T}} P \hat{e}+\hat{e}^{\mathrm{T}} P t \varepsilon \leq \beta^{2} \varepsilon^{T} \varepsilon+\frac{1}{\beta^{2}} \hat{e}^{\mathrm{T}} P t t^{\mathrm{T}} P \hat{e}$, then Eq.20 can be written as

$$
\dot{V} \leq \frac{1}{2} \hat{e}^{\mathrm{T}}\left(P T+T^{\mathrm{T}} P-\gamma^{\mathrm{T}} t^{\mathrm{T}} P-P t \gamma+\frac{1}{\beta^{2}} P t t^{\mathrm{T}} P+4 Q-2 \tau I\right) \hat{e}-\hat{e}^{\mathrm{T}} Q \hat{e}+\frac{1}{2} \beta^{2} \varepsilon^{T} \varepsilon
$$

For Eq. 21, if $P T+T^{\mathrm{T}} P-\gamma^{\mathrm{T}} t^{\mathrm{T}} P-P t \gamma+\frac{1}{\beta^{2}} P t t^{\mathrm{T}} P+4 Q-2 \tau I<0$, then $\dot{V} \leq-\hat{e}^{\mathrm{T}} Q \hat{e}+\frac{1}{2} \beta^{2} \varepsilon^{T} \varepsilon$. By Schur complement, $\left(\begin{array}{ccc}\Sigma & t & Z \\ * & -\beta^{2} I & 0 \\ * & * & -(4 Q-2 \tau I)^{-1}\end{array}\right)<0, \quad \Sigma=T z+Z T^{\mathrm{T}}-t \tilde{\gamma}-\tilde{\gamma}^{\mathrm{T}} t^{\mathrm{T}}, \tilde{\gamma}=\gamma P^{-1}, Z=P^{-1}$, “*” for the matrix block of symmetrical matrix. In addition $\dot{V} \leq-\hat{e}^{\mathrm{T}} Q \hat{e}+\frac{1}{2} \beta^{2} \varepsilon^{T} \varepsilon \leq-\frac{1}{2} \hat{e}^{\mathrm{T}} Q \hat{e}+\frac{1}{2} \beta^{2} \varepsilon^{T} \varepsilon$. Integrate it from $t=0 \quad$ to $\quad t=t_{\mathrm{f}} \quad, \quad$ considering $\quad V(t)>0 \quad, \quad \int_{0}^{t_{\mathrm{f}}} \hat{e}^{\mathrm{T}} Q \hat{e} d t \leq 2 V(0)+\beta^{2} \int_{0}^{t_{\mathrm{f}}} \varepsilon^{T} \varepsilon d t \quad$ and $V(0)=\frac{1}{2} \hat{e}(0)^{\mathrm{T}} P \hat{e}(0)+\frac{1}{2 \alpha} \tilde{\xi}^{T}(0) \tilde{\xi}(0)$. We can derive $\int_{0}^{t_{\mathrm{f}}} \hat{e}^{\mathrm{T}} Q \hat{e} d t \leq \hat{e}^{\mathrm{T}}(0) P \hat{e}(0)+\frac{1}{\alpha} \tilde{\xi}^{T}(0) \tilde{\xi}(0)+\beta^{2} \int_{0}^{t_{f}} \varepsilon^{T} \varepsilon$. It can be seen easily, the above inequality is just the $H_{\infty}$ tracking performance of Eq.11. [9,10,11]

\section{Simulation}

Consider the following control problem for macro gas turbine. The dynamic equations are as $T_{R}-T_{\text {load }}=J \frac{\mathrm{d} N}{\mathrm{~d} t}, T_{R}=\frac{1.16 \omega_{f}-0.87}{N}$. Let $\bar{N}=\frac{1}{2} N^{2}$, we have $\frac{\mathrm{d} \bar{N}}{\mathrm{~d} t}=N \frac{\mathrm{d} N}{\mathrm{~d} t}$ for the derivation of time with both sides, then we have $\frac{\mathrm{d} \bar{N}}{\mathrm{~d} t}=1.16 \frac{\omega_{f}}{J}-\left|\frac{1}{J}\left(1.414 T_{\text {load }} \sqrt{\bar{N}}+1.54\right)\right|$, where fuel flow $\omega_{f}$ is the input signal of the system, rotor speed $\mathrm{N}$ the output signal, $T_{R}$ the output torque of the rotor, $T_{\text {load }}$ the load torque, $\mathrm{J}$ the moment of inertia of the gas turbine rotor and $10 \mathrm{~kg} \cdot \mathrm{m}$.

External interference $\mathrm{d}(\mathrm{t})=$ rand- $0.5, d(t) \in L_{2}(0, \infty)$; The function rand will give a white noise with mean 0.5 , variance 0.5 . Reference input signal is the square wave. Let $\mathrm{N}=\mathrm{x} 1,{ }^{\omega_{f}}=\mathrm{x} 2$. The Gauss 
membership functions are chosen for variables $\mathrm{x} 1, \mathrm{x} 2$. Choose $\tau=0.15, \beta=0.03, \alpha=80$ and let $k_{\mathrm{r}}=\left(k_{\mathrm{r}, 2}, k_{\mathrm{r}, 1}\right)^{\mathrm{T}}=(1,2)^{\mathrm{T}}$. Control law and adaptive law of parameter $\Theta_{u}$ are as follows, respectively.

$$
u_{l}=\mu^{T}(x) \xi+(541.5324 e+364.4266 \dot{e}) / G(x) \quad \dot{\xi}=(22.048 e+11.8 \dot{e}) G(x) \mu(x)
$$

The $\mathrm{H} \infty$ rotation speed control performance is shown in Figs. 1 and Fig. 2. The curves of output $\mathrm{N}$ and the reference input ym are given in Fig. 1; the Fig. 2. shows the curve of the rotation speed error e of the gas turbine control system. The results of simulation illustrated that the rotation speed control system of GT has achieved the desired control performance.

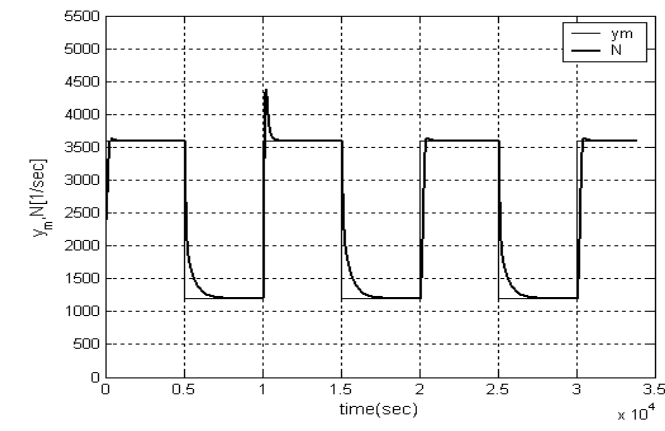

Figure 1. Rotation speed $N$ and reference input $y_{\mathrm{m}}$

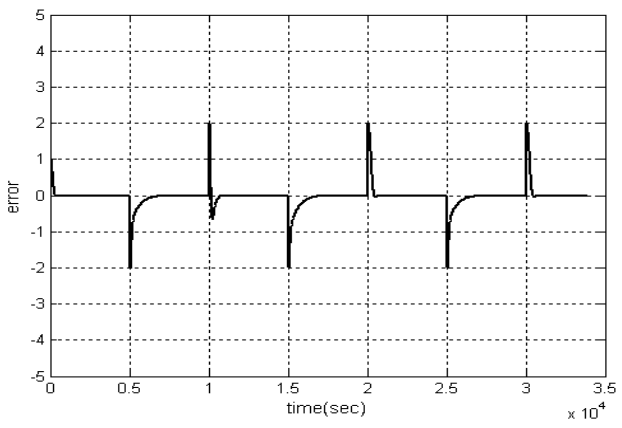

Figure 2. Curve of error $e$

\section{Conclusions}

The nonlinear control methods based on model cannot solve various control problems on a gas turbine control. Based on Lyapunov stability theory, a robust adaptive fuzzy $H_{\infty}$ control strategy on the basis of fuzzy basis functions is developed for the nonlinear system by means of linear matrix inequality technique. Simulations have demonstrated that all the states and the signals in the closed-loop system are all bounded. System rotation speed error can converge to a small neighborhood of the origin. For the given prescribed parameter, control system has achieved $H_{\infty}$ performance.

This proposed controller consists of three parts. The first part is an adaptive fuzzy controller equipped with parameters update laws. The second part is a variable structure control compensator to offset the effect of plant uncertainties and the third part is a robust controller to compensate the effect of the approximation error of fuzzy systems and external disturbance on rotation speed error.

\section{References}

[1] M. K. Passino, S. Yurkovich: Fuzzy control (Addison Wesley Longman, Inc., British 1998), p46.

[2] B. Wu, J. L. Song: Electric Machines and Control , Vol.17 (2013), No.5, p.98.

[3] Y. S. Yang: Information Science, Vol. 170 (2005), p.211.

[4] X. Ning, J. F. Chen and L. Q. Lu: Transactions of China Electrotechnical Society, Vol. 30 (2015), No.1, p.53.

[5] S. C. Tong, T. Wang and J. T. Tang: Fuzzy Sets and Systems, Vol.111 (2000), No.2, p.169.

[6] S. Reza, R.Mohammad and T. Akbarzadeh: IEEE Transactions on Fuzzy Systems, Vol. 16 (2008) No.3, p.187.

[7] C. S. Tseng, B. S. Chen and H. J. Uang: Fuzzy Sets and Systems, Vol. 9 (2001), No.3, p.381.

[8] H. G. Guo, B. J. Zhang: International Journal of Automation and Computing, Vol. 11 (2014), No. 4, p.418.

[9] Y. S. Yang: Information Science, Vol.170 (2005), p.211. 
[10] S. C. Tong, T. Wang and J. T. Tang: Fuzzy Sets and Systems, Vol.111 (2000), p.169.

[11] S. C. Tong, H. X. Li: Fuzzy Sets and Systems, Vol.128 (2002), No.1, p.107. 